\title{
Calibration of a fluorescence detector using a flying standard light source for the Telescope Array observatory
}

\section{Motoki Hayashi*}

Shinshu University, Nagano, Nagano, Japan

E-mail: 15tm524jeshinshu-u.ac.jp

\section{Yuichiro Tameda}

Kanagawa University, Yokohama, Kanagawa, Japan

E-mail: tame@kanagawa-u.ac.jp

\section{Yoshiki Tsunesada}

Tokyo Institute of Technology, Meguro, Tokyo, Japan

E-mail: tsunesadader.phys.titech.ac.jp

\section{Takayuki Tomida}

Shinshu University, Nagano, Nagano, Japan

E-mail: tomidadc.s.shinshu-u.ac.jp

\section{for the Telescope Array Collaboration ${ }^{\dagger}$}

The main calibration items of Fluorescence Detector (FD) in the Telescope Array (TA) observatory are the fluorescence yield, the atmospheric attenuation and the detector sensitivity. We are developing a flying standard light source which is called Opt-copter calibration system in order to calibrate the FDs. By mounting a high accurate global positioning system(GPS) device, the systematic error of the measured light source position is expected to be less than $1 \mathrm{~m}$. A photodiode mounted directly on the Opt-copter does measure the relative light intensity of each generating pulse. We report the progress of the Opt-copter development and the result of its test performance at the TA site.

The 34th International Cosmic Ray Conference,

30 July- 6 August, 2015

The Hague, The Netherlands

\footnotetext{
* Speaker.

${ }^{\dagger}$ For full author list and acknowledgments see http://www.telescopearray.org/images/papers/ICRC2015authorlist.pdf
} 


\section{Introduction}

The TA observatory performs the observation of ultra high energy cosmic rays (UHECRs) with energy above $10^{18} \mathrm{eV}$ using hybrid technique with fluorescence detectors (FDs) and surface detectors (SDs) array in Utah, U.S [四. The FDs measure calorimetric energy of cosmic rays by the detection of fluorescence UV lights emitted from Nitrogen molecules excited by high energy particles of an extensive air shower.

Moreover the FDs measure longitudinal development of the air shower, which is very important to identify a primary particle type of the UHECR. For the FD energy measurement, the calibration of detection efficiency is quite important. According to recent reports of the energy spectrum by the TA and the Pierre Auger Observatory (PAO) []][]], the energy scales can be different between the two experiments. Though the shape of energy spectrums is comparable upon energy shift correction, this energy scale difference must be solved in order to investigate the energy spectrum of the UHECR.

The energies observed by separate two FD stations at the TA site are also different to the level of $4 \pm 2 \%$ that is consistent within a systematic error. It is difficult to separate the FD detection efficiency and the atmospheric transparency effect to clarify the energy scale. On the site, the FDs are calibrated by the light sources, YAP and Xe flasher, mounted on each FD [䧃]. However, the FD efficiency cannot be compared directly by individual FD's standard light source. Thus, it is very important to calibrate by a common standard light source which is easy to move like a radio-controlled helicopter.

The FD alignment measurement is also important for the reconstruction of longitudinal development measured by the FD, because longitudinal development is the most effective information to study mass composition of the UHECR, especially the depth at shower maximum, $X_{\max }$. The alignment of TA FD was checked with tracks of stars in the field-of-view (FOV). However, by this method, enough stars might not be counted at low elevation region. For uniform check of all over the FD alignment, it is favorable to put a point light source in active. In order to get over above two

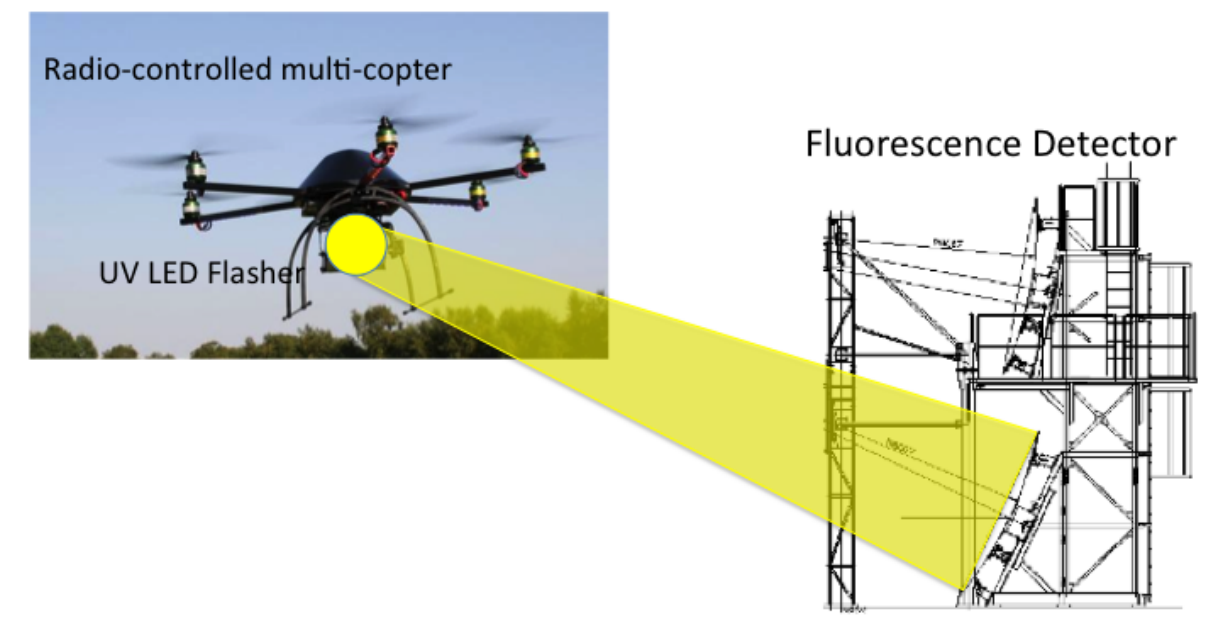

Figure 1: Schematic view of FD calibration by a flight light source. The light source is installed on a radio-controlled helicopter and tracking in the field of view of FDs. 
points, We are developing a movable standard light source. Figure $\mathbb{W}$ is the schematic view of FD calibration with a movable light source. The TA FD shown on the right side of Figure $\mathbb{\square}$ consists of a composite mirror and a photoMultiplier tube (PMT) cluster. The $3.3 \mathrm{~m}$ diameter mirror is composed of 18 hexagonal segments which have a focal length of $3.0 \mathrm{~m}$ and a spot size of $30 \mathrm{~mm}$ on the focal plane. The PMT cluster, installed on the focal plane, is composed of 256 hexagonal PMTs. Each PMT pixel views $\sim 1^{\circ}$ of the sky and the FD has a field of view of $15^{\circ}$ in elevation and $18^{\circ}$ in azimuth, respectively. The elevation angle of the upper FD is from $3^{\circ}$ to $18^{\circ}$ and that of the lower FD is from $18^{\circ}$ to $33^{\circ}$. The movable standard light source shown on the left side of Figure $\square$ consists of a radio-controlled helicopter and a UV LED flasher that is based on the same concept developed at Karlsruhe Institute of Technology (KIT) [[]]. On the TA site, we have already tested the light source [四].

In this report, we describe the specification and status of the movable standard light source development and the test performance at the TA FD site.

\section{Opt-copter}

The Opt-copter is the movable optical calibrator of the FD mounted on a octocopter which is radio-controlled helicopter with 8 rotors. It consists of a radio-controlled octocopter, a UV LED flasher, a high accurate GPS, a temperature monitor and a light intensity monitor. The flight of the Opt-copter can be controlled by the programed GPS information. The FDs detect the UV LED flash while the Opt-copter is flying into the FOV of each FD. By comparing the measured data and the ray-trace simulation, we perform calibrating FD's efficiency or measuring its alignment. It is very important for FD calibration with the Opt-copter in high accurate in order to pin down the atmospheric effect, because the LED lights pass through the atmosphere to the FD. The extinction coefficients at the TA site are comprised of molecular component $\alpha_{M}=0.060 \pm 0.002 \mathrm{~km}^{-1}$ and aerosol component $\alpha_{A}=0.033+0.016 /-0.012 \mathrm{~km}^{-1}$, under the satisfactory condition. Transmitted light intensity $I$ is defined upon the propagation length $x$ to the FD when $I_{0}$ is the initial light intensity at the light source. When the propagation length is $100 \mathrm{~m}$, the transparency is $99.1 \%$ and the systematic error is $-0.13 /+0.16 \%$. Thus the atmospheric transparency can be negligible when the Opt-copter is controlled within $100 \mathrm{~m}$ from the FDs.

\subsection{UV LED Flasher}

The UV LED flasher consists of 12 high power LEDs (H2A1-H375) and a spherical diffuser. The LED output power with a pulse width of $10 \mu \mathrm{s}$ and an electric current of $350 \mathrm{~mA}$ is $55 \mathrm{~mW}$ and the peak position of its wavelength spectrum is $375 \mathrm{~nm}$. This flasher is required to be uniform. The emission angle of each LED are $\pm 75^{\circ}$ and the LED is arranged on a regular dodecahedron. In order to improve the uniformity, we cover the LEDs with a spherical diffuser. The uniformity is $3 \%$ with the diffuser.

Moreover, it is also a key point to monitor the light intensity with high accuracy. The LEDs have temperature dependence, thus we should monitor temperature. Under the constant temperature, the stability of the LED intensity is to be less than $1 \%$. However, actual measurement condition was not stable. We plan to monitor the light intensity with photodiode on the LED flasher. 

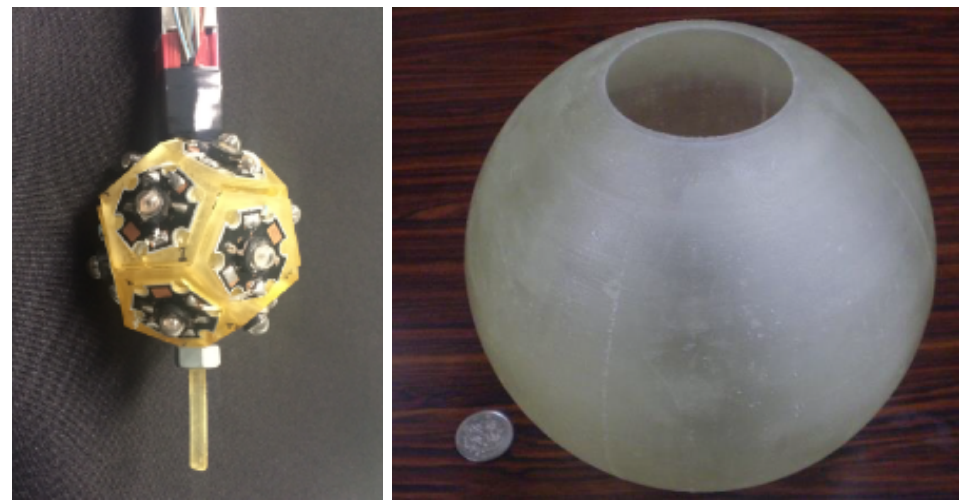

Figure 2: (Left) LEDs mounted on a regular dodecahedron. (Right) A diffuser, in which the LED mounted is set.

\subsection{Octocopter}

The radio-controlled octocopter (MK octo 2, Mikrokopter) is used to mount the LED flasher as shown in Figure [1. The octocopter with 8 rotors does fly very stably even if one of the rotors stops and can be controlled within $1.5 \mathrm{~km}$ away from the FD with programmed position information by the GPS. Payload capability of $2.0 \mathrm{~kg}$ during each flight of $20 \mathrm{~min}$. is enough to carry the LED flasher. The accuracy of the flight distance is less than $3 \mathrm{~m}$, but it is not enough for our purpose. Thus we need high accuracy positioning system of which accuracy is $\sim 10 \mathrm{~cm}$.

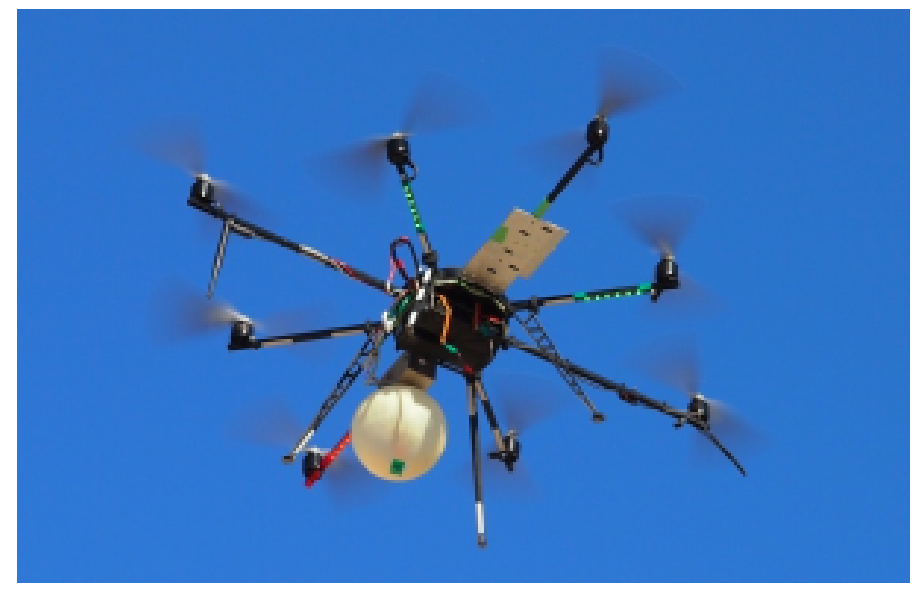

Figure 3: Octocopter : a radio-controlled helicopter with 8 rotors. At the bottom of the octocopter, the LED flasher is mounted.

\subsection{High Accuracy Positioning system}

High accuracy GPS receivers (Piksi, swift navigation) are used for the Opt-copter flight position measurement. One of the GPS receivers is set on the flight starting point and the other is mounted on the Opt-copter. This system measures the position with Real Time Kinematics (RTK) functionality to the centimeter level of relative positioning accuracy. Therefore the GPS system could reduce the systematic errors of the light intensity and the alignment measurements to less than $0.2 \%$ and $0.1^{\circ}$, respectively. 
At the TA site, we performed the test of the high accuracy positioning system. One of the GPS receivers is fixed as a base and the other is mounted on the Opt-copter as a rover. The base is located on the surveyed monument that is already measured with high accuracy of $\sim 2 \mathrm{~cm}$. The test result with single positioning mode is shown in Table $\mathbb{W}$. The differences of longitude and latitude are $0.5 \mathrm{~m}$ and $0.9 \mathrm{~m}$, respectively, which are consistent with the single positioning mode. On the other hand the difference of height is larger because the accuracy of height is 10 times larger than the case of latitude or longitude.

Table 1: The comparison of GPS information measured by Piksi and the surveyed monument. Piksi measured the position with single positioning mode.

\begin{tabular}{l|r|r|r}
\hline & Base measurements & Surveyed monument & Differences \\
\hline Latitude & $39^{\circ} 11^{\prime} 18.1170^{\prime \prime} \pm 0.0006$ & $39^{\circ} 11^{\prime} 18.09784^{\prime \prime}$ & $0.5 \mathrm{~m}$ \\
\hline Longitude & $-112^{\circ} 42^{\prime} 45.4124^{\prime \prime} \pm 0.0008$ & $-112^{\circ} 42^{\prime} 45.44244^{\prime \prime}$ & $0.9 \mathrm{~m}$ \\
\hline Height & $1402.10 \pm 0.04$ & 1395.080 & $7 \mathrm{~m}$ \\
\hline
\end{tabular}
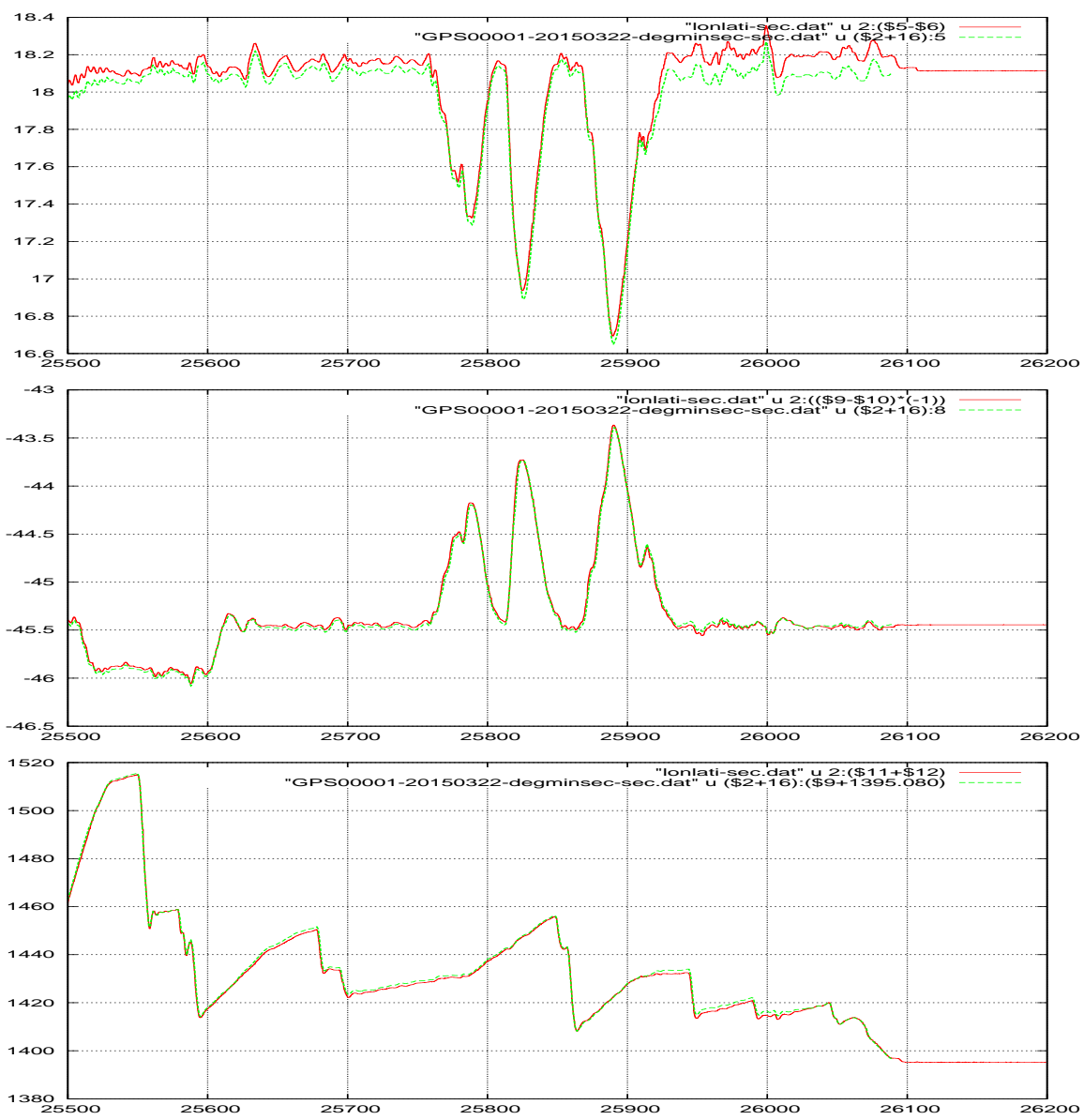

Figure 4: Track of the Opt-copter flight. Red and Green lines are measured by RTK mode and by MKGPS, respectively. MKGPS is used for flight control. Top, middle and bottom figures are latitude, longitude and height, respectively.

We also tested the accuracy of RTK measurement with Piksi at Shinshu University. Fixing the 
base, we put the rover next to the base and moved the rover, and measured the relative position between the base and the rover. The difference of relative position of RTK measurement is less than $4 \mathrm{~cm}$ on average for both cases. While the rover is static, the standard deviation of the rover position is less than $1 \mathrm{~cm}$, and the maximum difference is less than $6 \mathrm{~cm}$. The accuracy of RTK measurement was almost achieved accordingly. Figure $\$$ shows tracks measured by RTK mode and the MKGPS which is also mounted on the octocopter to control the flight. Each track is consistent within the flight accuracy of $3 \mathrm{~m}$.

\section{Test observation}

We performed the test for the Opt-copter during FD observation on March 22 and 24, 2015. We have succeeded to detect the Opt-copter signal by the FDs in normal observation mode, shown in Figure [5. However, at this flight, the light intensity is higher than our expectation of $\sim 10^{11}$ photons. The attenuation by diffuser and driver circuit was not enough and we plan to improve them.

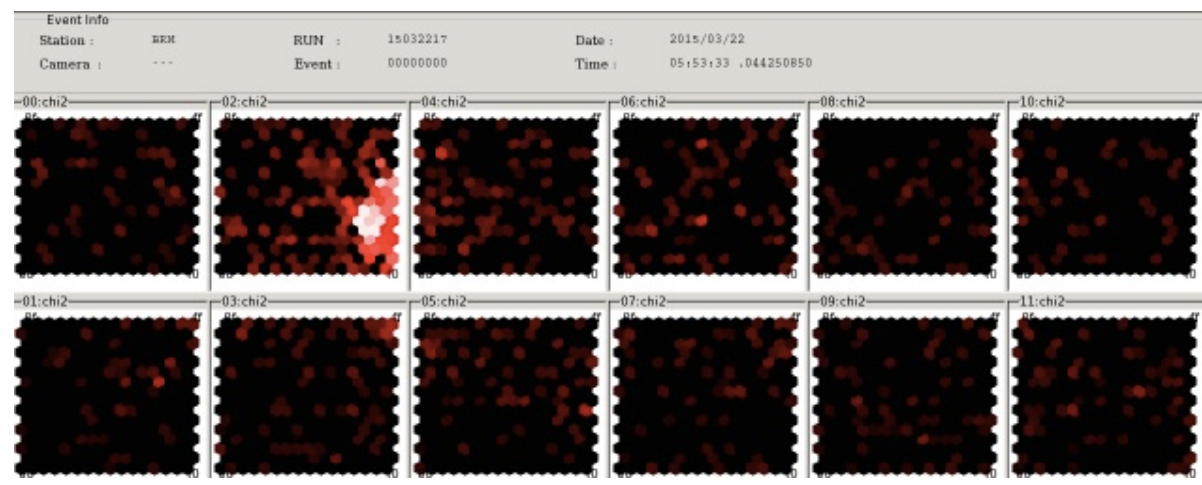

Figure 5: Event display of the optocopter signal observed by the TA FD.

\section{4. summary}

We are developing the Opt-copter, movable standard light source, in order to calibrate the FDs. We tested the high accuracy positioning system of RKT measurement and achieved enough accuracy. Stability and isotropy of the UV LED light source are measured as $<1 \%$ and $3 \%$, respectively. We tested the Opt-copter at the TA site on March 2015 and succeeded to observe the Opt-copter signal by the FDs. At present we are improving the diffuser and driver circuit to optimize the operation within $100 \mathrm{~m}$ away from the FD because the attenuation of the light intensity by the diffuser is insufficient to fit in the ADC dynamic range. In the future, by using the Opt-copter we will compare the detection efficiency of the FDs directly although the FDs are located separately. In addition We can also expect to apply the Opt-copter to calibrating various optical detectors installed outdoors. 


\section{Acknowledgments}

The Telescope Array experiment is supported by the Japan Society for the Promotion of Science through Grants-in-Aid for Scientific Research on Specially Promoted Research (21000002) "Extreme Phenomena in the Universe Explored by Highest Energy Cosmic Rays" and for Scientific Research (19104006), and the Inter-University Research Program of the Institute for Cosmic Ray Research; by the U.S. National Science Foundation awards PHY-0307098, PHY-0601915, PHY-0649681, PHY-0703893, PHY-0758342, PHY-0848320, PHY-1069280, PHY-1069286, PHY1404495 and PHY-1404502; by the National Research Foundation of Korea (2007-0093860, R3210130, 2012R1A1A2008381, 2013004883); by the Russian Academy of Sciences, RFBR grants 11-02-01528a and 13-02-01311a (INR), IISN project No. 4.4502.13, and Belgian Science Policy under IUAP VII/37 (ULB). The foundations of Dr. Ezekiel R. and Edna Wattis Dumke, Willard L. Eccles, and George S. and Dolores Doré Eccles all helped with generous donations. The State of Utah supported the project through its Economic Development Board, and the University of Utah through the Office of the Vice President for Research. The experimental site became available through the cooperation of the Utah School and Institutional Trust Lands Administration (SITLA), U.S. Bureau of Land Management, and the U.S. Air Force. We also wish to thank the people and the officials of Millard County, Utah for their steadfast and warm support. We gratefully acknowledge the contributions from the technical staffs of our home institutions. An allocation of computer time from the Center for High Performance Computing at the University of Utah is gratefully acknowledged.

\section{References}

[1] H. Tokuno, et. al., NIM A, 676, 54-65 (2012); T. Abu-Zayyad et. al., NIM A, 689, $87-97$ (2012)

[2] R.U. Abbasi et. al., Astropart. Physics, 68, 27-44 (2015)

[3] J. Abraham et. al., Phys. Letters B685 239 (2010)

[4] H. Tokuno, et. al., NIM A, 601, 364-371 (2009)

[5] F. Werner, Master's thesis, KIT (2010)

[6] K. Machida, et. al., Proc. of 33rd ICRC (2013) 